FedUni ResearchOnline

http://researchonline.federation.edu.au

This is the peer-reviewed version of the following article:

Vamplew, P., et al. (2017) Steering approaches to Pareto-optimal multiobjective reinforcement learning. Neurocomputing Vol. 263, p. 26-38.

Which has been published in final form at:

https://doi.org/10.1016/j.neucom.2016.08.152

Copyright () 2017 Elsevier B.V. All rights reserved. 


\title{
Steering Approaches to Pareto-Optimal Multiobjective Reinforcement Learning
}

\author{
Peter Vamplew ${ }^{\mathrm{a}}$, Rustam Issabekov ${ }^{\mathrm{a}}$, Richard Dazeley ${ }^{\mathrm{a}}$, Cameron Foale ${ }^{\mathrm{a}}$, \\ Adam Berry $^{\mathrm{b}}$, Tim Moore ${ }^{\mathrm{b}}$, Douglas Creighton ${ }^{\mathrm{c}}$ \\ ${ }^{a}$ Federation Learning Agents Group, School of Engineering and Information Technology, \\ Federation University Australia, Ballarat, Victoria, Australia \\ ${ }^{b}$ Energy Technology Division, CSIRO, Mayfield West, NSW, Australia \\ ${ }^{c}$ Centre for Intelligent Systems Research, Deakin University, Waurn Ponds, Victoria, \\ Australia
}

\begin{abstract}
For reinforcement learning tasks with multiple objectives, it may be advantageous to learn stochastic or non-stationary policies. This paper investigates two novel algorithms for learning non-stationary policies which produce Pareto-optimal behaviour (w-steering and Q-steering), by extending prior work based on the concept of geometric steering. Empirical results demonstrate that both new algorithms offer substantial performance improvements over stationary deterministic policies, while Q-steering significantly outperforms w-steering when the agent has no information about recurrent states within the environment. It is further demonstrated that Q-steering can be used interactively by providing a human decision-maker with a visualisation of the Pareto front and allowing them to adjust the agent's target point during learning. To demonstrate broader applicability, the use of Q-steering in combination with function approximation is also illustrated on a task involving control of local battery storage for a residential solar power system.
\end{abstract}

Keywords: multiobjective reinforcement learning, non-stationary policies, geometric steering, interactive reinforcement learning, Pareto optimality

\section{Introduction}

Reinforcement learning (RL) methods learn the optimal behaviour for 3 an agent on the basis of a reward signal received from the agent's environ4 ment. While most RL research assumes the agent has only a single objective 
to be maximised, many sequential decision-making tasks exhibit multiple conflicting objectives. Examples include managing competing uses of water reservoirs [1], reducing energy usage and increasing miss probability detection in cognitive radio networks [2], achieving both optimal control and stability in a control system [3], and considering both engagement time and transition smoothness in wet clutch control [4]. Therefore recent years have seen multiobjective reinforcement learning (MORL) emerge as a growing area of research. For an overview of this field see [5].

The difference between single-objective RL and MORL is straightforward - rather than a scalar reward, the agent receives a vector reward with an element for each objective. For MORL problems multiple Pareto-optimal policies may exist, and the agent must either learn several of these policies, or identify a single policy which best matches a pre-specified tradeoff between the objectives. It may be that no deterministic stationary policy closely matches the desired trade-off, and so non-stationary or stochastic policies may be superior. However there has been relatively little work so far on MORL algorithms using such policies.

This paper addresses this limitation by investigating two novel algorithms for learning non-stationary policies for multiobjective problems, w-steering and Q-steering ${ }^{1}$. These algorithms adapt the Multiple Direction Reinforcement Learning (MDRL) steering algorithm of $[7,8]$ to the task of learning Pareto-optimal policies. w-steering is a direct extension of MDRL, while Q-steering incorporates a heuristic policy-selection mechanism to address MDRL's reliance on pre-existing knowledge of the identity of recurrent states of the environment. The performance of these two approaches to Paretooptimal steering are empirically compared, demonstrating Q-steering's superior performance across a variety of problems.

This paper then examines two extensions of Q-steering. Section 7 proposes an interactive approach to MORL, by providing a human user with a visualisation of the Pareto front learnt by Q-steering, and allowing them to iteratively modify the agent's behaviour. Section 8 demonstrates the applicability of Q-steering to real-world problems with high-dimensional state-space

\footnotetext{
${ }^{1}$ Some preliminary results related to these algorithms were presented in [6]. This paper provides an extended justification for the design of these algorithms, and an enhanced experimental evaluation of the factors affecting their performance, as well as considering extensions of the Q-steering algorithm to interactive MORL and learning in state spaces requiring function approximation.
} 
by using Q-steering in conjunction with function approximation to control local battery storage for a photovoltaic power system.

\section{Background}

Single-objective RL generally assumes that the environment can be modelled as a Markov decision process (MDP) defined by the following variables:

- $S$ is a finite set of states,

- $A$ is a finite set of actions,

- $T: S \times A \times S \rightarrow[0,1]$ is a state transition function specifying for each state, action, and next state, the probability of that next state occurring,

- $R: S \times A \times S \rightarrow \Re$ is a reward function, which defines the expected immediate reward for each state, action and subsequent state,

- $\mu: S \rightarrow[0,1]$ is a probability distribution over initial states, and

- $\gamma \in[0,1)$ is a discount factor specifying the relative importance of shortterm and long-term rewards.

The goal of the agent is to find a policy (a mapping from state to action) which maximizes the expected return $R_{t}$. In a finite horizon (or episodic) $\mathrm{MDP}$, the return is typically an undiscounted finite sum of the rewards over all time-steps until a terminating state is reached, while in an infinite horizon MDP, the return is usually an infinite sum, with each term discounted according to $\gamma$ :

$$
R_{t}=\sum_{k=0}^{\infty} \gamma^{k} r_{t+k+1},
$$

where $r_{t}$ is the reward obtained at time $t$.

In multiobjective MDPs (MOMDPs) the reward function $\mathbf{R}: S \times A \times S \rightarrow$ $\Re^{n}$ is a vector of $n$ rewards rather than a scalar, with an element for each objective. Similarly, the return is also a vector value. For example, the multiobjective infinite horizon return $\mathbf{R}_{t}$ would be defined as:

$$
\mathbf{R}_{t}=\sum_{k=0}^{\infty} \gamma^{k} \mathbf{r}_{t+k+1},
$$

For single objective MDPs there will be either a single optimal policy, or a set of optimal policies with identical returns. For MOMDPs a policy can 

be divided into two broad categories:
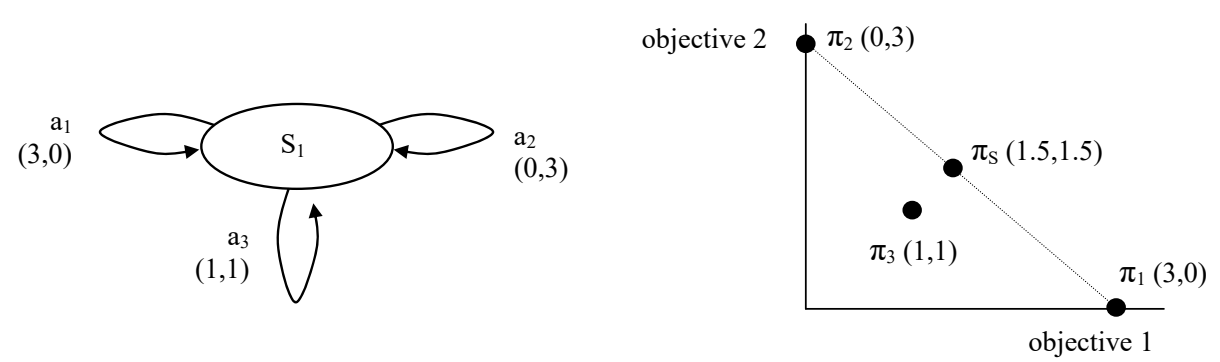

outperform another on one objective, but be inferior with regards to the other objective(s). This is summarized by the Pareto dominance relationship. A policy dominates another if it is superior on at least one objective, and at least equal on all other objectives. Clearly a dominating policy is preferable to the policies it dominates. However there may be many policies which are non-dominated - all such policies may be optimal, depending on the desired trade-off between the objectives. $[9,5]$ propose that MORL algorithms can

- multi-policy approaches that learn multiple policies which approximate the complete Pareto front, or a subset of that front; or

- single-policy approaches that learn a single policy which best satisfies a desired trade-off between objectives expressed in advance.

As well as differentiating between multiple optimal policies, a MORL agent may need to consider forms of policies which are not required in a single-objective context. For this work we consider the following policy types:

- deterministic stationary (DS) policies always select the same action when faced with a particular state of the environment. The subset of DS policies which can be found via linear scalarisation (see Section 3) is also relevant - we will refer to these as linear deterministic stationary (LDS) policies,

- stochastic policies in which the choice of action is non-deterministic, and

- non-stationary policies in which the choice of action in any state is nonstationary with regards to the state, and is conditioned on some other variable, such as the rewards previously received by the agent.

Figure 1: An MOMDP with 3 actions, labeled with their rewards. The graph shows average per-action reward for each deterministic stationary policy $\pi_{1}, \pi_{2}$ and $\pi_{3}$ and for a stochastic policy $\pi_{s}$ which selects actions $a_{1}$ and $a_{2}$ with equal probability.

77 For fully-observable single-objective MDPs there is always a DS pol78 icy which is optimal [10]. However this may not be true for MOMDPs, 
where stochastic or non-stationary policies may dominate the best DS policies $[11,12]$. Consider the single-state MOMDP in Figure 1. Only three DS policies exist, all of which are non-dominated. If a balanced trade-off between objectives is required then policy $\pi_{3}$ which always selects $a_{3}$ will be the best option. However consider a stochastic policy $\pi_{s}$ which selects actions $a_{1}$ and $a_{2}$ with probabilities $p_{1}$ and $\left(1-p_{1}\right)$ respectively. The average reward of this policy will be $\left(3 p_{1}, 3-3 p_{1}\right)$. If $1 / 3 \leq p_{1} \leq 2 / 3$ then this policy will dominate the DS policy $\pi_{3}$. This simple example illustrates the general point that for multiobjective problems it may be possible to generate superior results using policies which are either non-stationary or stochastic.

This section has outlined the general manner in which MORL differs from the more traditional single-objective case. For the remainder of this paper it is assumed that the MORL agent must identify a single Pareto-optimal policy that satisfies the user's preferred trade-offs between objectives, and that it will exploit the benefits of stochastic or non-stationary policies. In addition it is assumed that the algorithm must be well-suited to online learning, and that it will not require any prior knowledge of the MOMDP's state transition or reward dynamics. The implications of these requirements will be explored in the next two sections which review existing approaches to MORL with stochastic and non-stationary policies.

\section{Learning Stochastic or Non-Stationary Multiobjective Policies}

The MORL literature has explored two main approaches to learning nonstationary or stochastic policies. The first uses policy search methods which directly produce stochastic policies, such as policy-gradient methods [10,13] or multiobjective evolutionary algorithms $[14,15]$. Policy search methods may have advantages for MORL, as they can be applied in conjunction with non-linear scalarisation functions to specify desired trade-offs between objectives [5]. However comparisons of policy search and value-based singleobjective RL indicate that policy search algorithms may converge more slowly and so be less suitable for on-line learning, particularly if state transitions or rewards are stochastic. $[16,17,18]$. As such there may be situations in which a value-based approach to stochastic or non-stationary MORL is preferable.

The main value-based approach explored is to find DS policies, and then to form stochastic or non-stationary combinations of these 'base policies' $[7,8,12,19]$. An advantage of this approach is that the base policies can be found using simple methods. For example, linearly scalarised temporal 
difference learning has been widely used to find LDS policies for MORL tasks $[20,21,22]$. Linear scalarisation takes a weighted sum of the rewards, converting the problem to a single-objective MDP so standard TD-based methods can be used [5]. Repeated runs with differing weights can discover a range of Pareto-optimal policies $[1]^{2}$. The agent then switches between base policies, either stochastically [12] or on a non-stationary basis $[7,8]$. An advantage of these methods is that once the base policies have been learnt they can readily be combined to produce different stochastic or nonstationary policies with no further sample costs. This paper extends the work of $[7,8]$, and so the next section will discuss their approach in more detail.

\section{The Geometric Steering Approach to MORL}

[7] proposed and analysed an MORL algorithm based on the concept of geometric steering. [8] provided a more detailed presentation and formally named it Multiple Directions Reinforcement Learning (MDRL). MDRL is designed for stochastic games and so assumes that the environment may also be influenced by the actions of another agent. However [7] note that MOMDPs can be treated as a special case of this multiobjective stochastic game model. We will restrict our discussion of MDRL to the MOMDP scenario.

As outlined in Algorithm 1, MDRL forms a non-stationary policy conditioned on prior rewards, which aims to keep the average reward vector within a defined target region in objective space. The agent learns in parallel multiple base policies based on linear scalarisation with different weight vectors. At any time a single base policy is active and the agent 'steers' the average reward towards the target region by switching between base policies. The choice of active policy is based on a direction vector from the average reward vector obtained so far to the closest point in the target region. The policy whose weights best match the direction vector is then selected as the active policy (see lines 8 and 9 of Algorithm 1). The intuition here is that following this policy will drive the average reward vector towards the target region.

MDRL has a number of desirable properties. The desired trade-off between objectives is specified in a straight-forward fashion via the bounds

\footnotetext{
${ }^{2}$ As demonstrated by [23] linear scalarisation is limited to discovering policies which lie on the convex hull of the Pareto front (the Convex Coverage Set or CCS [19]). However in the context of learning base policies this is actually advantageous as these CCS policies are in fact sufficient to construct all optimal stochastic or non-stationary polices $[12,19]$.
} 
of the target region. In addition, steering can be viewed as a flexible "metaalgorithm' as the learning of base policies can be achieved using any methods based on linear scalarisation of the objectives - while the results reported in this paper are based on Q-learning, any off-policy algorithm could be used. Steering can also be applied in non-learning contexts, to form a combination of base policies found via multiobjective planning methods such as $[19,24]$.

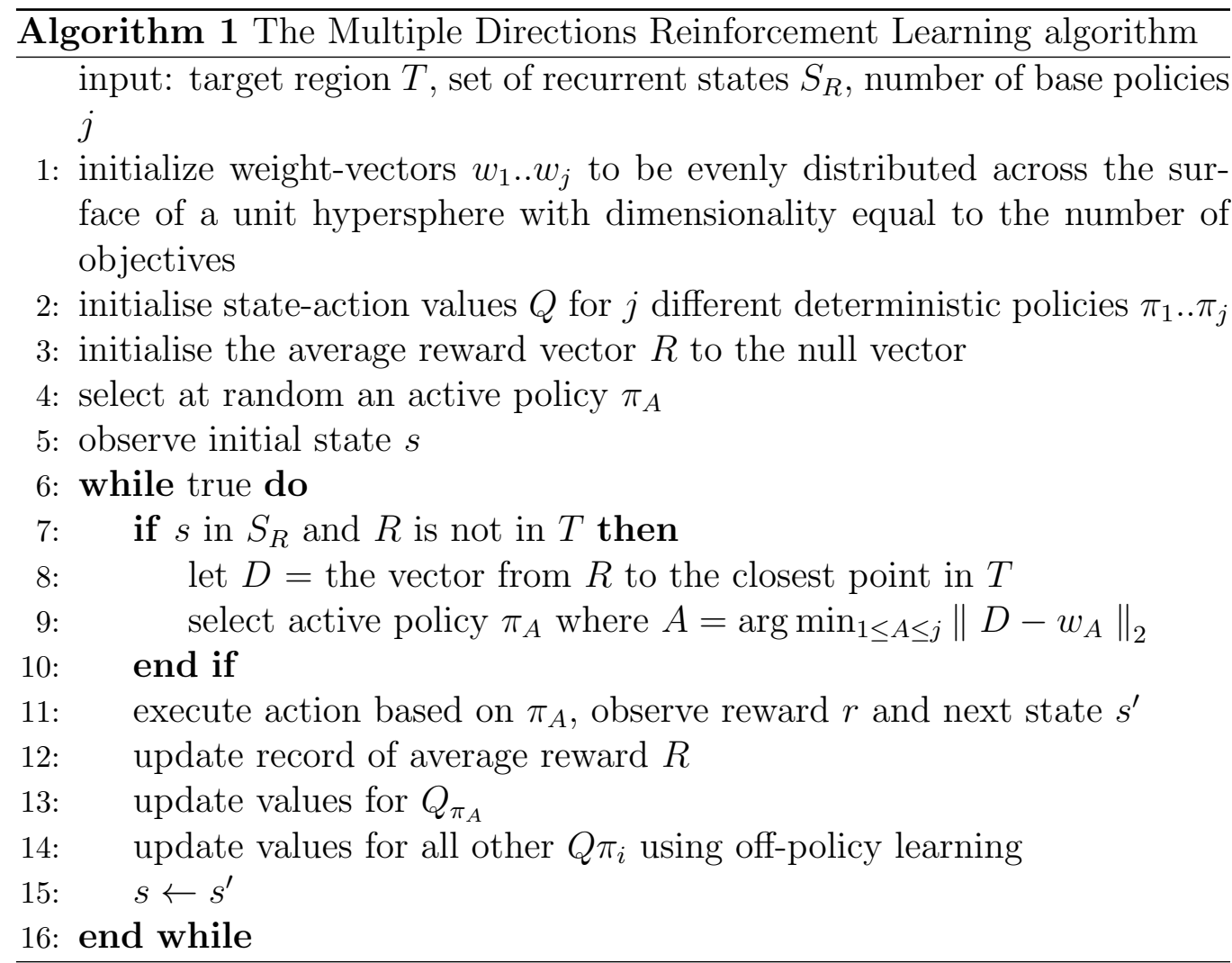

However MDRL relies on two key assumptions. It is assumed that one or more states of the environment are recurrent - that is, they are guaranteed to be revisited within a finite period of time regardless of the policy followed by the agent. Formally, if the hitting time of a state $s^{*}$ is defined as $\tau_{s^{*}}=\min \left\{n>0: s_{n}=S^{*}\right\}$ and $E_{\pi}^{s}$ is the expectation operator when starting from state $s_{0}=s$ and following policy $\pi$, then there must exist at least one state $s^{*}$ and a finite constant $N$ such that $E_{\pi}^{s}\left(\tau_{s^{*}}{ }^{2}\right)<N$ for all $\pi \in \Pi$ and $s \in S$. In addition it is assumed that the agent knows the identity of these recurrent states and only switches policies when in one of these states, 
as indicated by the set $S_{R}$ in Algorithm 1. This requires prior knowledge of the environment's state dynamics. For some problems this may be easy - for example in episodic problems the initial state of each episode can be treated as recurrent. However more generally there may be insufficient prior knowledge to identify these states, and then the only viable option is for MDRL to allow switching in any state, which could lead to suboptimal performance ${ }^{3}$.

[8] address this limitation in an alternative algorithm known as Current Direction Reinforcement Learning (CDRL). CDRL maintains a single policy, which has scalarisation weights set to match the current direction from the average reward vector to the target set. This policy is followed for an extended period of time before considering whether to switch policy by altering the weights. Switching can occur in any state, and so CDRL does not require $S_{R}$ as an input. By following a single policy for an extended period, CDRL reduces the frequency of policy switching which will minimise, but not eliminate, the potential impact of switching policies in inappropriate states. However while CDRL will maintain the long-term average reward within the target region, in the short-term the average reward may lie well outside this region. For many applications this increase in the variance of the short-term reward will be unacceptable. For this reason we will not consider CDRL further in this work. Instead the next section will explore two variations of the MDRL approach to steering, including one specifically designed to address the problems introduced by lack of information about recurrent states.

\section{Applying Steering to Pareto-Optimal MORL}

While MDRL provides a means for learning non-stationary policies for MOMDPs, there are several differences between the context for which it was originally designed, and the context which this work addresses. Most significantly, MDRL treats the vector rewards as criteria to be satisfied rather than as objectives to be maximised. As shown in Figure 2 the specified target region of reward space may include both lower and upper bounds for each reward. For example the scenario in [7] requires the agent to keep the environmental temperature and humidity within a bounded region. The agent may need to steer in both positive and negative directions in reward space (as shown by vectors $D_{1}$ to $D_{4}$ ). Therefore the agent must identify

\footnotetext{
${ }^{3}$ The alternative is to treat no states as recurrent, but this would mean the agent would never switch base policies, thereby losing the benefits of non-stationarity.
} 
not just the Pareto-optimal policies $\left(\pi_{1}\right.$ and $\left.\pi_{2}\right)$ but also the dominated policies with rewards lying on the other side of the target region $\left(\pi_{3}\right.$ and $\left.\pi_{4}\right)$. To achieve this, the weight vectors $w_{1} . . w_{j}$ must include both positive and negative weights. In contrast when the goal is to produce Pareto-optimal behavior (as in Figure 3), the use of negative weights is neither required nor appropriate.

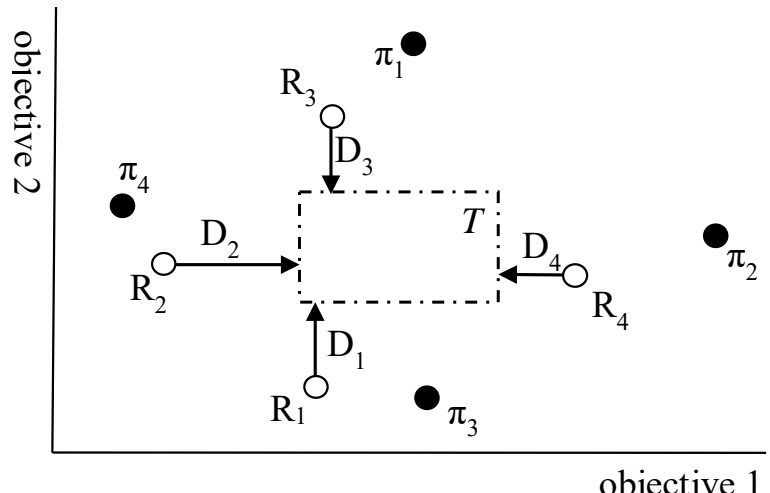

Figure 2: Illustrating MDRL. $T$ is the target region, the white dots $R_{1}$ to $R_{4}$ indicate possible points for the average reward so far, and the arrows $D_{1}$ to $D_{4}$ indicate the corresponding direction vectors to the nearest point in $T$. The black dots $\pi_{1}$ to $\pi_{4}$ indicate the base policies which may be followed by the agent.

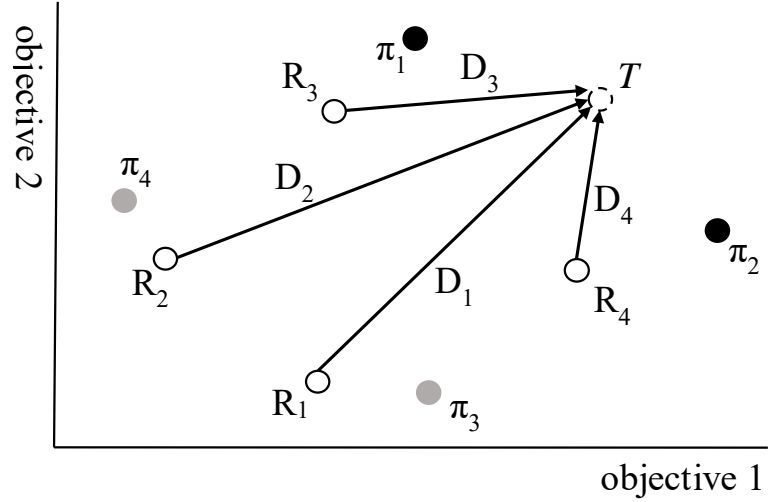

Figure 3: Pareto-optimal steering. $T$ is the target point, the white dots $R_{1}$ to $R_{4}$ indicate possible points for the average reward so far, and the arrows $D_{1}$ to $D_{4}$ indicate the direction vectors to $T$. In contrast to Figure 2 the agent will only require the Pareto-optimal base policies $\pi_{1}$ and $\pi_{2}$.

Similarly in the context of Pareto optimality, there may be no pre-existing knowledge on which to base the size or shape of the target region, so we propose to use a target point in objective space instead, as shown in Figure 
3. Specifying a target point is intuitive for a human decision-maker, and by changing the target, the agent can be directed towards different Paretooptimal policies. In addition the user may interactively change the target during learning as they learn more about the achievable trade-offs between the objectives - this approach will be examined further in Section 7.

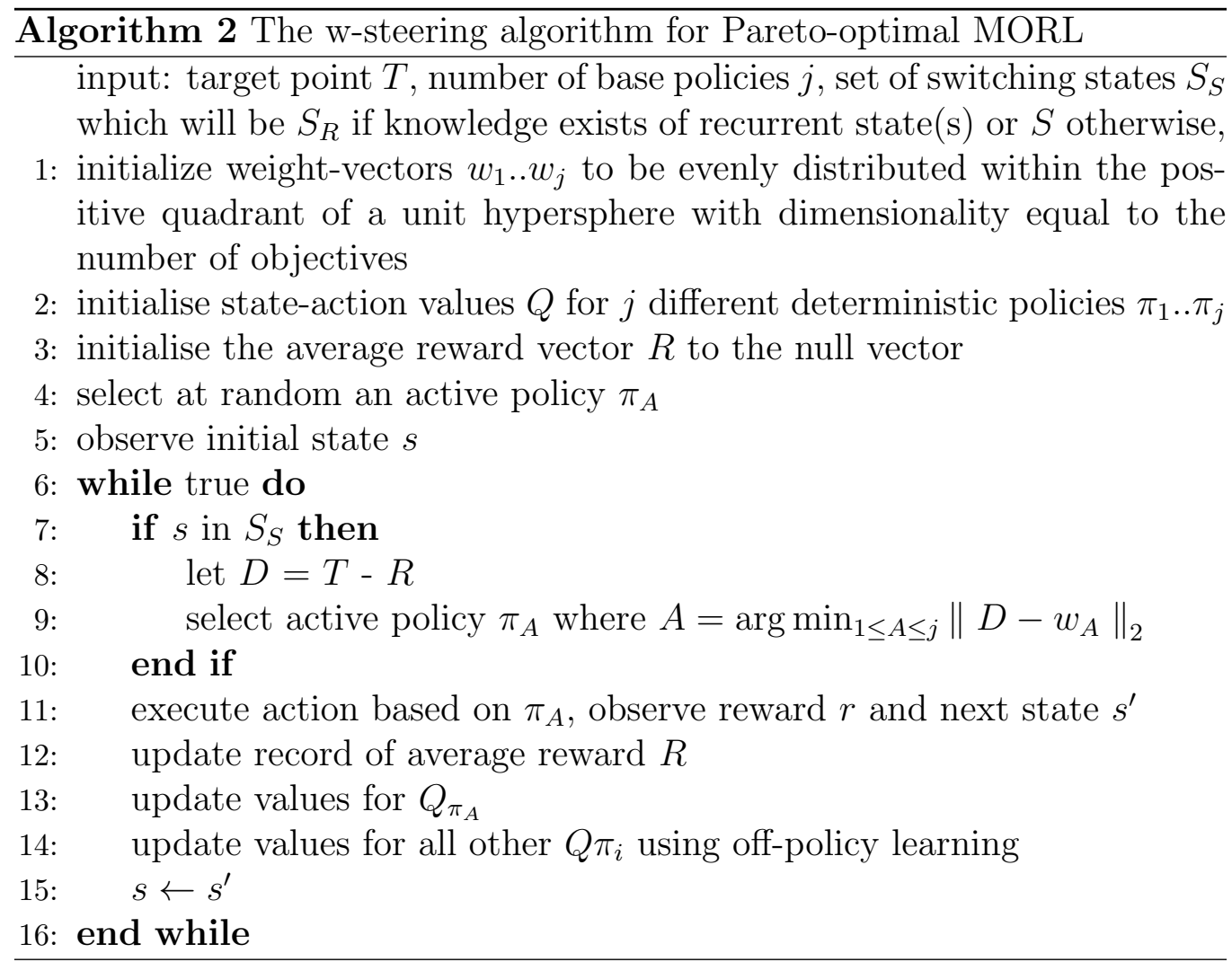

Algorithm 2 modifies MDRL to address the goal of finding a Paretooptimal policy. However this modified algorithm retains the fundamental assumption of MDRL - that the agent is provided with a set of states which are known to be recurrent under all policies. Both algorithms may perform poorly in the absence of such knowledge as their policy selection (line 9 of Algorithms 1 and 2) is based on the direction vector to the target and the weight vector of each base policy, and so is independent of the current state of the environment. This fails to take into account that certain states may be far more desirable for some base policies than for others - for example in the Linked Rings problem (Figure 6 in Section 6) states 2, 3 and 4 are desirable 
if maximizing the first objective, but undesirable if maximizing the second objective. We refer to this as the switching-state identification problem.

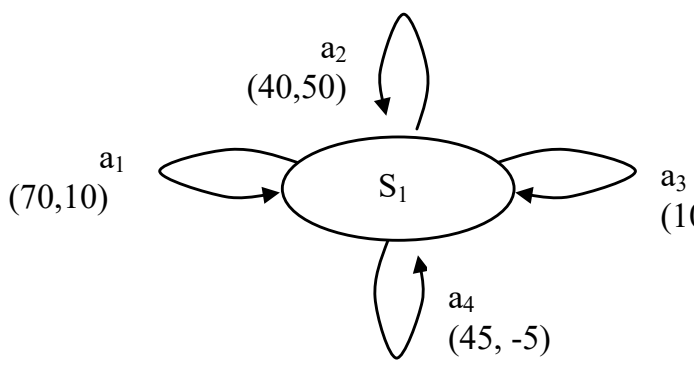

Figure 4: (left) A simple environment to illustrate a potential issue with weight-based policy selection as used in MDRL and Algorithm 2.

The MDRL approach to policy selection has a further potential flaw. Selecting the policy with weights that best align with the direction vector $D$ may not in fact result in moving the average reward closer to the target, due to the non-linear relationship between the weights and the return achieved by the policy [25]. Consider the example in Figure 4. If the base policy weights are $w_{1}=(1,0), w_{2}=(\sqrt{0.5}, \sqrt{0.5})$, and $w_{3}=(0,1)$, then three base policies $\pi_{1}, \pi_{2}$ and $\pi_{3}$ will be discovered corresponding to always selecting actions $a_{1}, a_{2}$ and $a_{3}$ respectively (action $a_{4}$ is Pareto-dominated by $a_{1}$ and so is not selected under any Pareto-optimal policy). Assume that the target is $(50,30)$, which can be achieved by alternating between $\pi_{1}$ and $\pi_{2}$. Also assume that due to exploration the agent has so far selected actions $a_{1}, a_{2}, a_{3}$ and $a_{4}$ in the ratio $7: 7: 2: 4$, giving rise to an average reward of $(48.5,26)$. The vector $D$ will be $(6.5,4)$, so the active policy at this point should favour the first objective over the second (i.e. $\pi_{1}$ ). However Algorithm 2 will select policy $\pi_{2}$ as its weight vector is the smallest distance from $D$. In the context of steering towards a large target region as in MDRL, this issue is less problematic however in the context of steering towards a target point, policy selection errors such as this will lead to behaviour which is not Pareto-optimal. We will refer to this as the policy identification problem.

We propose a modified Pareto-optimal steering algorithm to account for both of these issues with Algorithm 2, by basing policy selection not each policy's weights but on the expected value of each policy at the current state. As shown in lines 9-12 of Algorithm 3 the value of the greedy action is found for each base policy, and the policy whose value maximises the dot 
Algorithm 3 The Q-steering algorithm. Note that the only variation from Algorithm 2 is in the policy selection mechanism (lines 9 to 12).

input: target point $T$, set of switching states $S_{S}$ which will be equal to $S_{R}$ if knowledge exists of recurrent state(s) or equal to $S$ otherwise, number of base policies $j$

1: initialize weight-vectors $w_{1} . . w_{j}$ to be evenly distributed within the positive quadrant of a unit hypersphere with dimensionality equal to the number of objectives

2: initialise state-action values $Q$ for $j$ different deterministic policies $\pi_{1} . . \pi_{j}$

3: initialise the average reward vector $R$ to the null vector

4: select at random an active policy $\pi_{A}$

5: observe initial state $s$

6: while true do

7: $\quad$ if $s$ in $S_{S}$ then

8: $\quad$ let $D=T-R$

9: $\quad$ for each policy $\pi_{i}$ do

10: $\quad Q^{*}{ }_{i}=Q_{\pi_{i}}\left(s, a^{*}\right)$ where $a^{*}=\arg \max _{a} w_{i} \cdot Q_{\pi_{i}}(s, a)$

11: $\quad$ end for

12 :

13:

14: $s^{\prime}$

15: $\quad$ update record of average reward $R$

16: $\quad$ update values for $Q_{\pi_{A}}$

17: update values for all other $Q \pi_{i}$ using off-policy learning

18: $s \leftarrow s^{\prime}$

19: end while

Q-steering's policy selection approach acts as a heuristic to address the switching-state identification issue. States not visited under a particular policy $\pi$ will tend to have lower values for $Q_{\pi}\left(s, a^{*}\right)$, and so the agent will be less likely to select $\pi$ in those states. Instead switching will tend to occur in 
the recurrent states as these are visited under all policies. This heuristic also allows the agent to opportunistically switch policies should it find itself in a state which is particularly beneficial with regards to a specific objective [26]. This approach also addresses the policy-identification problem. By basing selection on the actual $Q_{\pi}\left(s, a^{*}\right)$ values, the policy that is best aligned to move the average reward towards the target will be selected. For example, in the case shown in Figure 4 Q-steering will correctly select policy $\pi_{1}$.

Q-steering and w-steering share a common implementation issue, regarding the updating of the average reward vector $R$. Using a cumulative average for $R$ would mean that poor performance on an objective early in learning would influence the value of $R$ and hence affect the agent's behaviour for an extended period of time. Conversely an exponentially weighted moving average may be biased early in learning by the values used to initialise $R$. The implementation used in these experiments uses a cumulative average initially, before switching to an exponentially weighted moving average. That is,

$$
\mathbf{R}_{t}= \begin{cases}\sum_{k=0}^{t} \mathbf{r}_{k} / t, & \text { if } t \leq L \\ \tau \mathbf{r}_{t}+(1-\tau) \mathbf{R}_{t-1}, & \text { otherwise }\end{cases}
$$

where $0<\tau<1$ and $L$ is a threshold time period. For non-episodic tasks $R$ is updated every time-step and $L$ is defined in time-steps, whereas for episodic tasks $R$ is updated at the end of an episode based on the accumulated rewards over that episode, and $L$ is defined in episodes.

\section{Empirical Evaluation of w-Steering and Q-Steering}

While $[7,8]$ provide an extensive theoretical analysis of MDRL, this relies on the assumptions which underpin MDRL and so is not directly applicable to w-steering and Q-steering. Therefore this section empirically compares the two variants of Pareto-optimal steering on three benchmark problems - one in which the set of recurrent states $S_{R}$ is known, one in which this information is not available to the agent, and one with no recurrent states.

\subsection{Benchmark problems}

The first benchmark is DST-Mixed (Figure 5), which is a variant of the Deep Sea Treasure task [23]. The agent controls a treasure-hunting subma-

rine, and must compromise between the value of the treasure and the time 

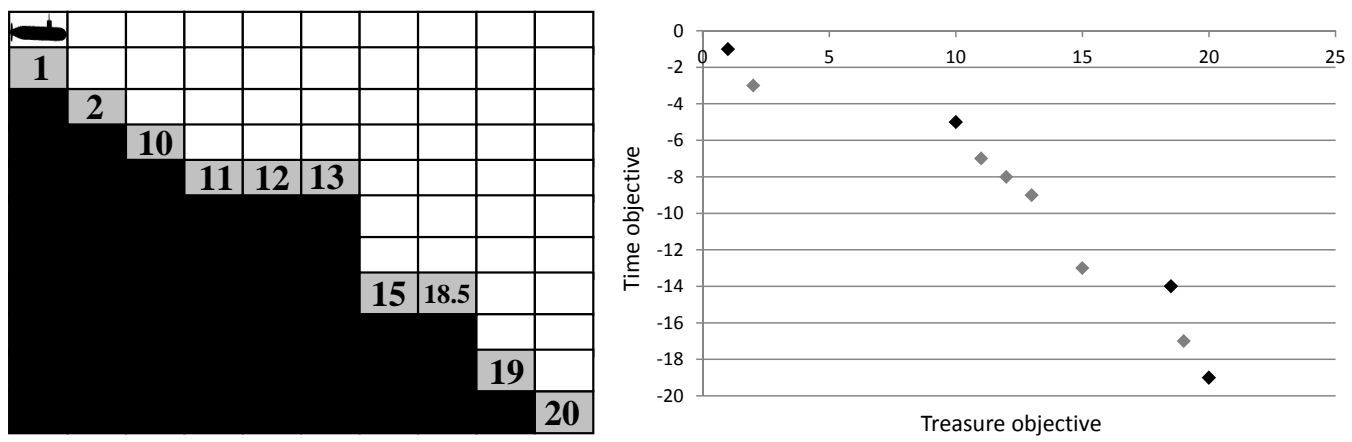

Figure 5: (left) The DST-Mixed environment. The agent starts each episode in the top, left cell marked with a submarine. (right) The Pareto front for the DST-Mixed task. Black points show policies which can be found by linear scalarisation (LDS policies).

taken to find it. A reward of -1 is given for the time objective on all timesteps in which a treasure is not found, while the treasure reward is zero except when a treasure is found at which point it is equal to the treasure value in the grey cells in Figure 5. The original DST has a concave Pareto front, such that only two LDS policies exist. As this does not adequately test the ability of the steering algorithms to select the correct base policy, the DST-Mixed variant has modified treasure values so that four LDS policies exist. This is an episodic task with a fixed starting state for all episodes and so that state can be used as the switching state for steering $\left(S_{R}=\left\{S_{\text {start }}\right\}\right)$.

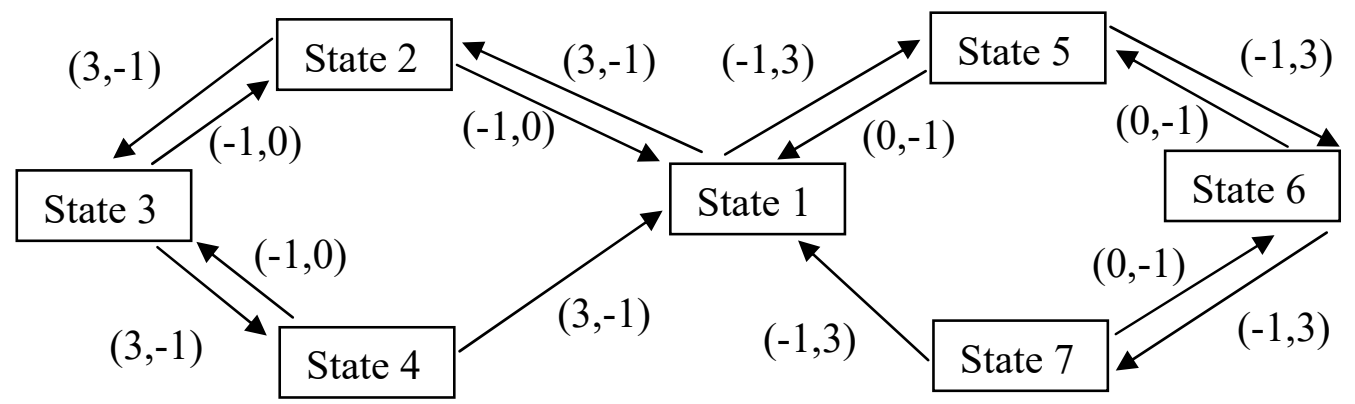

Figure 6: The Linked Rings benchmark problem.

The second benchmark is the non-episodic Linked Rings (Figure 6). Four Pareto-optimal DS policies exist (see Table 1). Only $A$ and $D$ are LDS policies, and so these form the base policies for steering. A maximises objective 1 and always moves counter-clockwise, whereas $D$ maximises objective 2 and always moves clockwise. Table 1 shows that State 1 is the only recurrent 


\begin{tabular}{l|l|l}
\hline Policy ID & $\begin{array}{c}\text { States visited when starting } \\
\text { in State } 1\end{array}$ & Average reward per action \\
\hline A & $\mathbf{1}, 2,3,4, \mathbf{1}, \ldots$ & $3,-1$ \\
$\mathrm{~B}$ & $\mathbf{1}, 2, \mathbf{1}, \ldots$ & $1,-0.5$ \\
$\mathrm{C}$ & $\mathbf{1}, 5, \mathbf{1}, \ldots$ & $-0.5,1$ \\
$\mathrm{D}$ & $\mathbf{1}, 5,6,7, \mathbf{1}, \ldots$ & $-1,3$ \\
\hline
\end{tabular}

Table 1: The four Pareto-optimal DS policies for the Linked Rings.

state and so is the correct state in which to switch between base policies, but it is assumed this information is not available to the agent, and so both steering algorithms will treat all states as switching states $\left(S_{S}=S\right)$. This benchmark is designed to examine the performance of the two algorithms when no prior knowledge is available about the correct switching states.

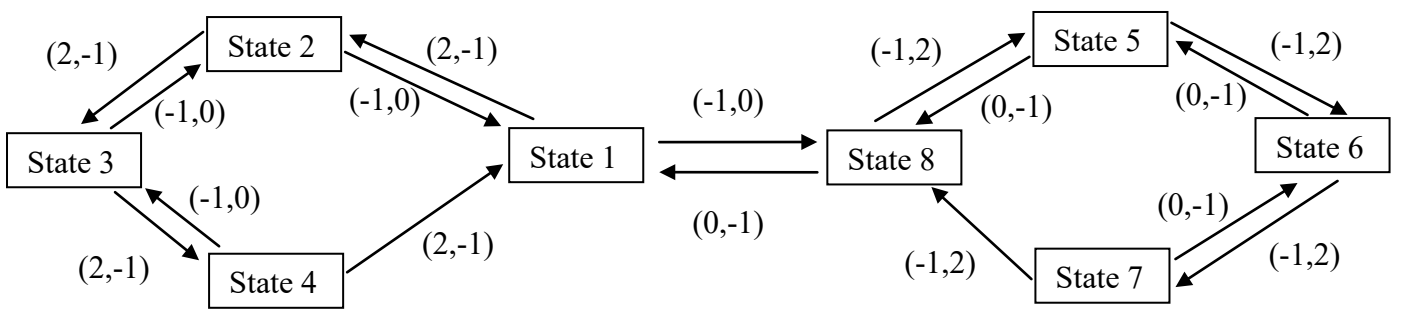

Figure 7: The Non-Recurrent Rings benchmark problem.

The final benchmark is the non-episodic Non-Recurrent Rings (Figure 7). This has a similar structure to the Linked Rings, but the addition of an extra state and arcs means that there is no state which is recurrent under all Pareto-optimal policies (see Table 2). This benchmark tests the ability of the algorithms to operate in an MOMDP without recurrent states. The optimal steering behaviour is to switch between base policies A and D only when in states 1 and 8, but this switching should occur less frequently than in the Linked Rings, as here there is a cost associated with switching policies due to the lower rewards on the arcs between states 1 and 8 .

\subsection{Experimental Method and Metrics}

The performance of a MORL algorithm may vary depending on where in the Pareto-front the desired solution lies - for example, an algorithm may be good at finding policies which focus on an individual objective, but poor for policies with equal tradeoffs between objectives [9]. For this reason a series 


\begin{tabular}{l|l|l}
\hline Policy ID & $\begin{array}{c}\text { States visited when starting } \\
\text { in State } 1\end{array}$ & Average reward per action \\
\hline $\mathrm{A}$ & $1,2,3,4,1, \ldots$ & $2,-1$ \\
$\mathrm{~B}$ & $1,2,1, \ldots$ & $0.5,-0.5$ \\
$\mathrm{C}$ & $1,8,5,8,5 \ldots$ & $-0.5,0.5$ \\
$\mathrm{D}$ & $1,8,5,6,7,8, \ldots$ & $-1,2$ \\
\hline
\end{tabular}

Table 2: The four Pareto-optimal DS policies for the Non-Recurrent Rings.

of different target points were generated for each of the benchmark problems, distributed at different locations along the convex hull of the Pareto front, thereby ensuring that the algorithms can provide sufficient coverage of the entire front [19]. To satisfy these convex-hull target points, a steering algorithm must be able to identify the correct combination of base policies, as well as the correct ratio with which to combine those policies.

We assume a decision-maker using a steering algorithm will not have knowledge of the exact front and so is unlikely to specify a target point precisely on the front. Therefore it is necessary to test the impact on the algorithm of specifying either a pessimistic target (one lying below the front), or an optimistic target (one lying above the front). Therefore additional sets of targets were identified by projecting different distances along a line passing through each of the convex-hull target points orthogonal to the slope of the hull. In all cases, if the agent learns the Pareto-optimal policy which minimises the distance to the specified target, then the average reward will be equal to the corresponding convex-hull target point. The target points for each benchmark are listed in Tables A.4, A.5 and A.6 in the Appendix.

For each target, w-steering and Q-steering were trained for 5000 episodes (DST-Mixed) or time-steps (Linked Rings and Non-Recurrent Rings). The $\epsilon$ parameter controlling exploration was linearly decayed to zero over this period. Once learning was completed, a further 100 episodes/time-steps were executed in a strictly greedy fashion, and the average reward over this period was recorded. The greedy policy was then evaluated by measuring the Euclidean distance between the average reward and the convex-hull target point matching the target given to the agent. The mean distance over each set of target points of the same degree of pessimism/optimism was then calculated. This process was repeated for ten independent trials, with the mean calculated over these trials. Tabular Q-learning was used as the underlying algorithm for finding base policies for both steering algorithms. Table 3 
summarises the parameter settings of both algorithms for each benchmark.

\begin{tabular}{l|l|l}
\hline Parameter & DST-Mixed & $\begin{array}{c}\text { Linked Rings and } \\
\text { Non-Recurrent Rings }\end{array}$ \\
\hline$\alpha$ (learning rate) & 0.9 & 0.9 \\
$\lambda$ (eligibility trace decay) & 0.95 & 0.95 \\
initial $\epsilon$ & 0.9 & 0.2 \\
$\gamma$ (discounting) & 1.0 & 0.9 \\
$j$ (number of base policies) & 8 & 4 \\
$\tau$ (decay term for averaging) & 0.99 & 0.99 \\
$L$ (time threshold for averaging) & 100 episodes & 100 timesteps \\
\hline
\end{tabular}

Table 3: The Q-learning and steering parameters. The number of base policies was set to twice the number of Pareto-optimal LDS policies for each benchmark to ensure that the weight-space sampling identified the required base policies.

\subsection{Experimental Results and Discussion}

Figure 8 shows the mean results for both steering algorithms on each of the benchmark tasks, across the range of optimistic, neutral and pessimistic targets. The best results theoretically achievable using DS and LDS policies are also shown. These were determined by selecting the DS or LDS policy whose average return most closely matches each target. The benefits of non-stationary policies are evident, with both steering algorithms producing results which are clearly superior to those achievable using DS or LDS policies. The only exception is the Non-Recurrent Rings where the absence of recurrent states causes w-steering to be outperformed by the best DS policies.

The behaviour of both steering algorithms is inhibited by the use of pessimistic targets, although the results achieved are still superior to LDS and, in most cases, DS policies. The performance of the steering algorithms is also quite stable across the range of neutral and optimistic target points, indicating that they are not overly sensitive to the degree of optimism.

On the DST-Mixed benchmark, both steering algorithms were able to closely match the provided target point. For optimistic targets Q-steering produced an exact match in most runs, while w-steering was slightly poorer. For this problem the agents knew the recurrent state, so these differences must be due to w-steering's policy identification problem. For the Linked Rings and Non-Recurrent Rings, policy identification is a less pressing concern as only two unique LDS policies exist. Therefore the improvements demonstrated by Q-steering on these tasks are due to its ability to handle 


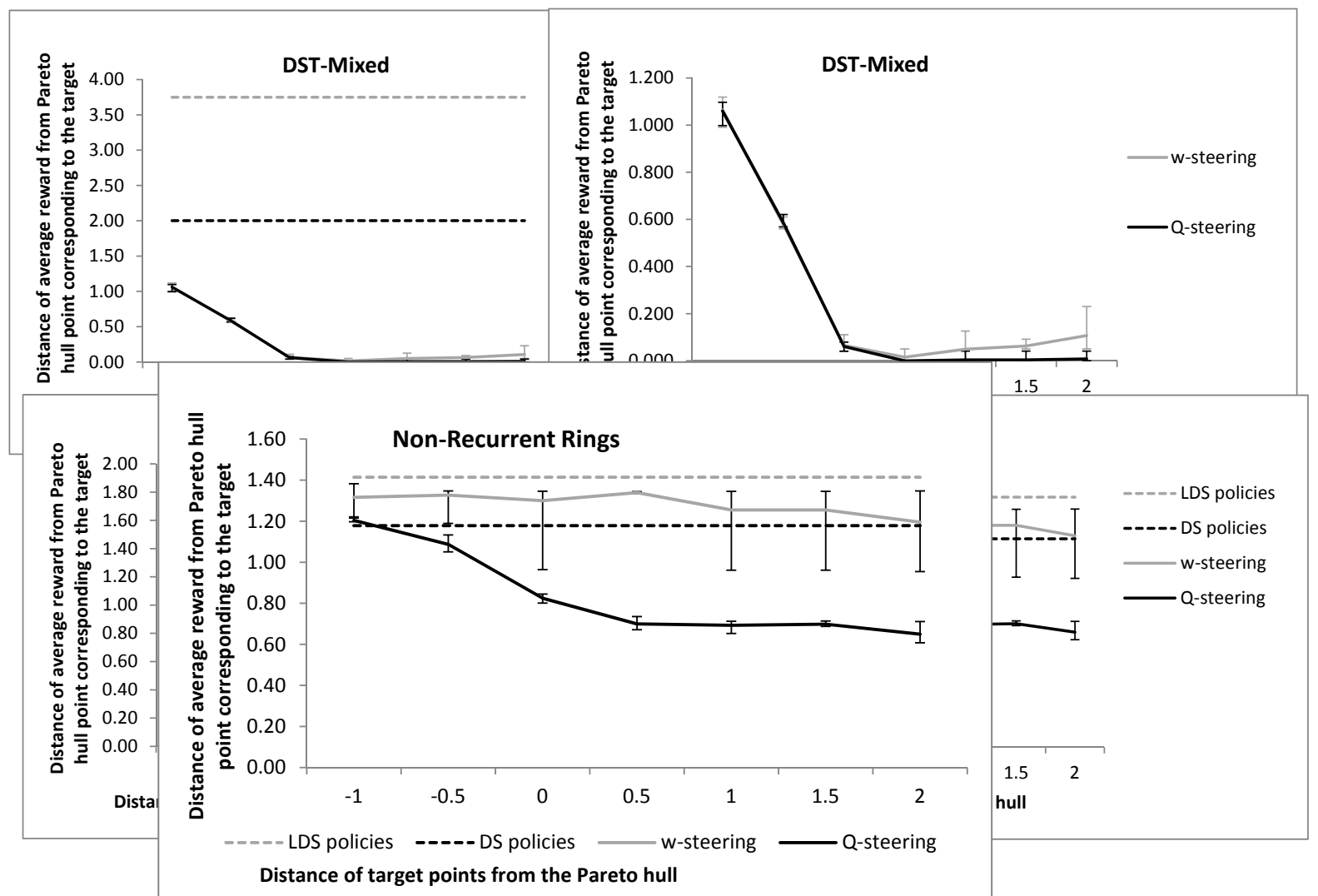

Figure 8: Mean distance of average reward from the Pareto hull point corresponding to the target over ten trials of w-steering and Q-steering compared to the best results possible using DS or LDS policies. Error bars show the best and worst result achieved in any trial. For DST-Mixed a zoomed view is also shown.

the switching-state identification problem. On the LinkedRings, Q-steering correctly identified state 1 as the optimal switching state whereas w-steering often switched policies in states 2 and 5, giving rise to sub-optimal returns. Similarly on the Non-Recurrent Rings, w-steering often switched policies in states other than 1 and 8, thereby producing sub-Pareto-optimal results, whereas Q-steering minimised these mistakes.

It should be noted that there was considerably more variation in performance on these tasks compared to DST-Mixed, as shown by the error bars in Figure 8. In particular it was observed that when the target strongly favoured one objective, Q-steering occasionally converged to policies which performed poorly on the less-favoured objective. This occurred in around $10 \%$ of runs, and did not occur at all when the target point was balanced between both objectives. This issue appears to be caused by the more fre- 
quent clearing of eligibility traces, and therefore slower learning, experienced by the non-active base policies. It may be that higher levels of exploration are required to ensure that all base policies are learnt correctly under these circumstances. Nevertheless, even in those runs Q-steering's performance was superior to that of the other methods.

In summary Q-steering has been shown to learn non-stationary policies which produce Pareto-optimal results which closely match the specified target. This represents a substantial improvement over w-steering, addressing both the switching-state identification and policy-identification problems of the latter algorithm. Q-steering's performance is also a major improvement over the results achievable using stationary deterministic MORL, with reductions in error with respect to the target of between 40 and 100\%. Q-steering's performance benefits hold regardless of whether the environment's recurrent states are known or not, or even in the case of the Non-Recurrent Rings task, where there are no recurrent states at all.

\section{Interactive Q-Steering}

The results in Section 6.3 demonstrate that Q-steering can produce Paretooptimal behaviour when provided with a suitable choice of target point, particularly if that target is slightly optimistic with regards to the Pareto front. For many problems, it should be straightforward for a human decision-maker to specify such a target to indicate their desired preference between objectives. For example, if MORL is being applied to a task where prior measures of performance of alternative methods such as human control exist, then those measures can be used as a guide when specifying a target.

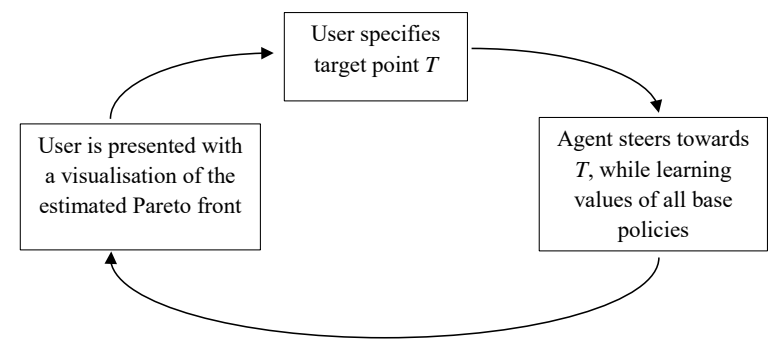

Figure 9: An abstract view of interactive Q-steering. The visualisation of the front is updated as the agent learns more accurate estimates of the value of each base policy, and is used to inform the user's choice of target point.

However in some cases there may be little information available to guide the user in specifying a target. It is also possible that the preferred trade-offs 
may vary over time as circumstances not captured in the environmental state change (for example, a coach of a Robocup team may instruct their agents to focus more on a defensive objective and less on scoring when defending a narrow lead late in a game). Q-steering can be applied to these scenarios by allowing the decision-maker to interactively change the target during or after learning. As the Q-steering agent has learnt base policies covering the entire Pareto hull, when the target changes it should be able to switch its choice of active base policies so as to steer towards the new target. To aid the user in making an informed choice of target, the agent should maintain an estimate of the Pareto-front and provide a visualisation of this to the user, who may then alter the specification of the target, as shown in Figure 9.

Interaction between a human user and an agent in the context of MORL has previously been explored by several authors. Most commonly this has been in the context of multi-policy learning for decision support, where the agent's role is to identify the possible range of behaviours available, with the final choice of trade-offs between objectives then made by the human $[19,5,27]$. In this scenario the interaction between the human and agent takes place only once learning has been completed, in contrast to interactive Q-learning where the human decision-maker can be involved throughout the learning process. This form of ongoing interaction has also previously been considered, most notably in work on preference-based RL (PBRL) [28, 29]. A PBRL agent learns in the absence of a numerical reward signal, on the basis of qualitative feedback provided by the human user in the form of preferences over either actions, trajectories or policies. As such it depends on continued interaction with the user throughout the learning process. [29] comment on the similarities which exist between PBRL and MORL - however, they also note that MORL can be seen as a special case of the PBRL framework in that a numerical reward signal is available, and that therefore algorithms which make use of that reward information (such as Q-steering) are likely to be more effective than general PBRL algorithms.

\subsection{Visualising the Estimated Pareto Front}

To visualise the front, an estimate is required of the average-reward value $P_{\pi_{i}}$ for each base policy $\pi_{i}$. These can then be displayed to the user in either graphical or tabular format. For problems with a single known recurrent state (such as DST-Mixed), $P_{\pi_{i}}$ can be approximated by the value of the greedy action for that state for that policy. More generally if $S_{R}$ contains multiple states (as in an episodic task with multiple starting states), then 
Algorithm 4 Deriving an estimate of the Pareto front from base policy Q-values

input: set of switching states $S_{S}$, set of known recurrent states $S_{R}$ (possibly empty), number of base policies $j$, state $\mathrm{s}, Q_{\pi_{i}}(s, a)$ for each base policy, active base policy $\pi_{A}$, discounting rate $\gamma$, time threshold $L$

1: if $s$ in $S_{S}$ then

2: $\quad$ for each policy $\pi_{i}$ do

3: $\quad$ if $\left(\pi_{i}=\pi_{A}\right)$ or $\left(s\right.$ in $\left.S_{R}\right)$ then

4: $\quad Q^{*}{ }_{i}=Q_{\pi_{i}}\left(s, a^{*}\right)$ where $a^{*}=\arg \max _{a} Q_{\pi_{i}}(s, a)$

5: $\quad$ if $t<L$ then

$6:$

$7:$

8:

9:

10:

11:

12:

$13:$

14:

$15:$

16 :

$$
17:
$$

Algorithm 4 details the complete process used to estimate the Pareto front points from the Q-values of each base policy. Note that $t$ is defined in 

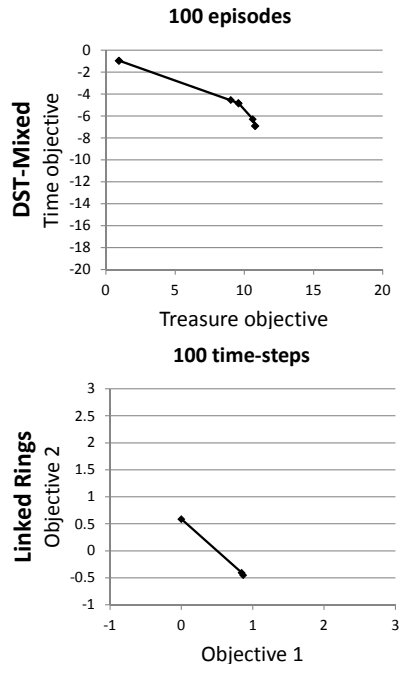

100 time-steps

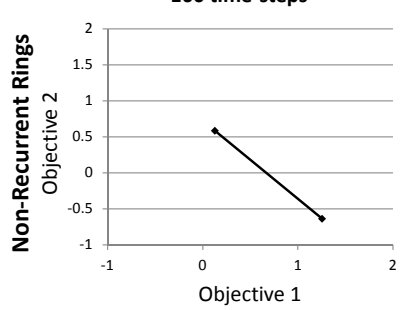

200 episodes
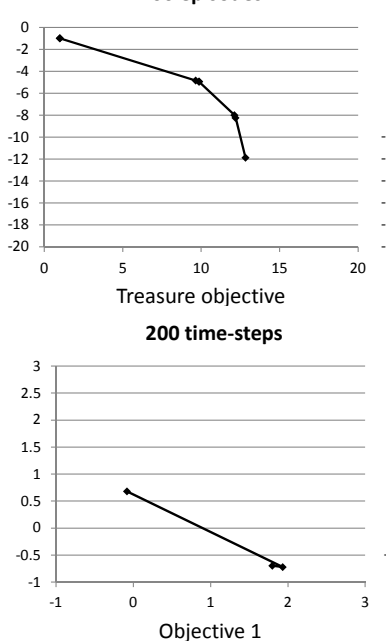

200 time-steps
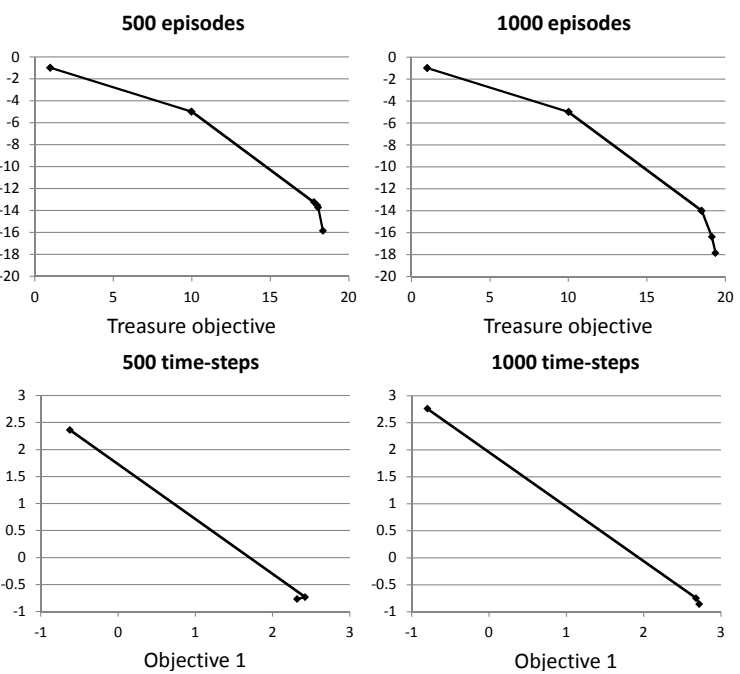

500 time-steps

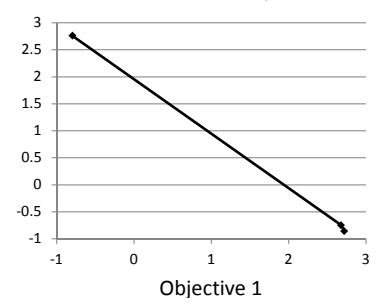

1000 time-steps
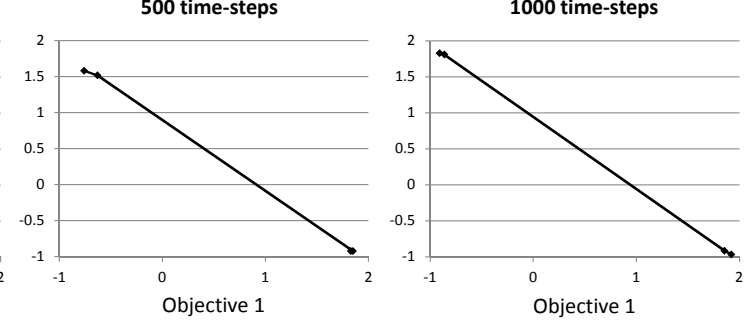

Figure 10: The estimated Pareto front produced at various points in learning for a randomly-selected run on each of the benchmarks.

To simulate the user interaction with Q-steering as described in the previous subsections, the following experimental methodology was applied:

- The target $T$ was initialised to an overly optimistic target $T_{I}$, based on the maximum value obtainable for each objective for that benchmark.

- Q-steering was executed with estimates of the Pareto front derived at regular intervals during learning using Algorithm 4.

- To simulate the user changing the target, after 2000 episodes or timesteps $T$ was changed to a final target point $T_{F}$, and learning continued. The value of $T_{F}$ was selected from the set of target points at distance 1 from the Pareto hull of the benchmark, as listed in Tables A.4, A.5 and A.6. 


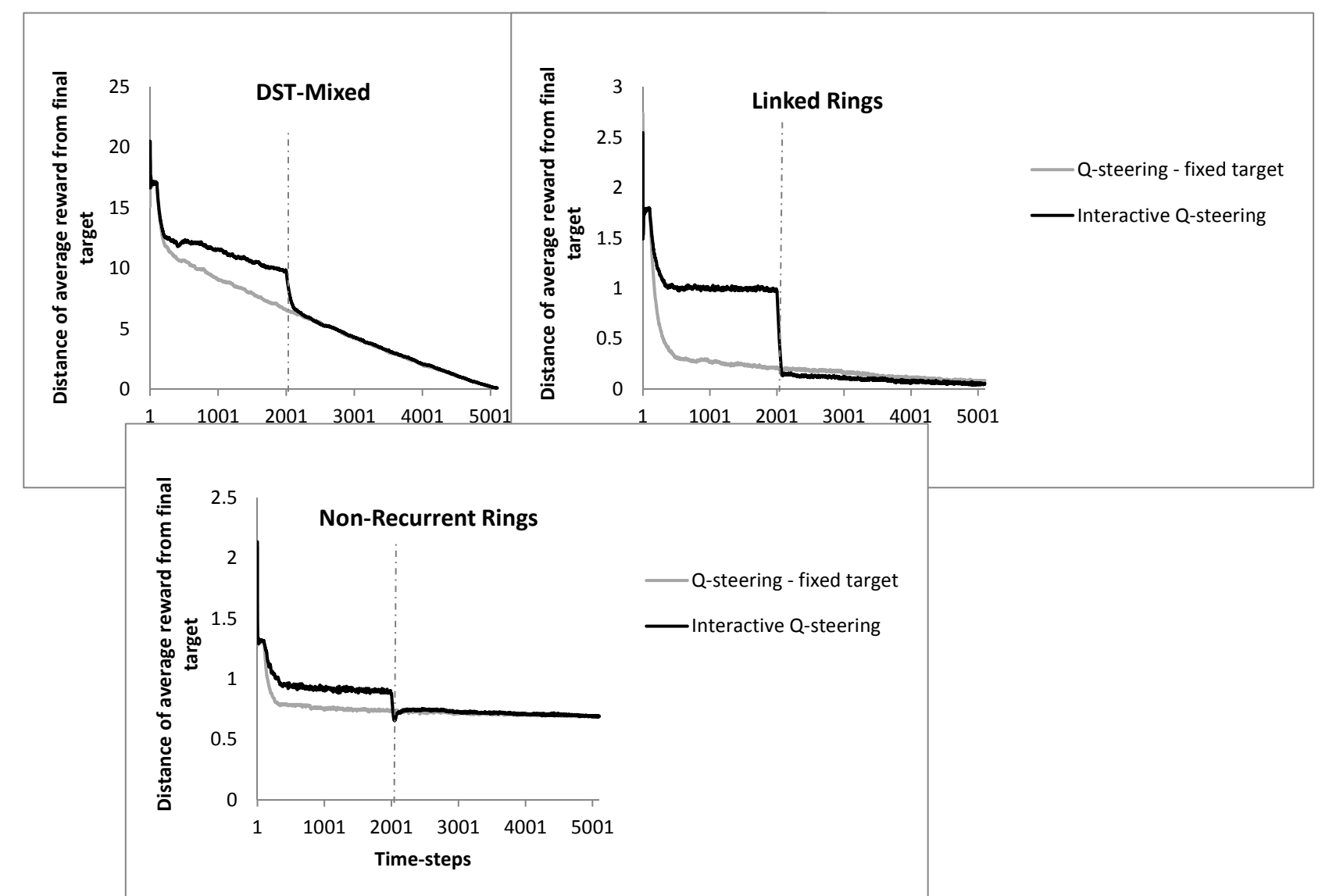

Figure 11: Mean distance of average reward from the Pareto hull point corresponding to the final target $T_{F}$ over ten trials for Q-steering with $T=T_{F}$ throughout training, and for interactive Q-steering where $T$ switches from $T_{I}$ to $T_{F}$ after 2000 episodes or time-steps. The dashed vertical line marks the stage at which the target was changed.

- This process was repeated 10 times for each target for each benchmark. The performance was measured in terms of the distance between the average reward $R$ and the Pareto hull point corresponding to target $T_{F}$.

As shown in Figure 10, the initial estimate of the front for each benchmark is poor, but improves rapidly as the agent learns. In all cases after 1000 timesteps or episodes the visualisation of the front is sufficiently accurate to allow the user to modify the target. Figure 11 shows that early in learning, the error with respect to the Pareto hull point corresponding to $T_{F}$ is quite high - this is expected as during this period the agent is trying to optimise its 
behaviour with regards to the initial target $T_{I}$. Once a suitable visualisation of the front is obtained and the target point $T$ changed to $T_{F}$ there is a sharp drop in error, as the agent immediately modifies its policy selection to satisfy this new target. In fact on the Linked Rings and Non-Recurrent Rings, the performance of the interactive agent with respect to $T_{F}$ is surprisingly slightly improved over that of a Q-steering agent using $T=T_{F}$ throughout training. This was due to the interactive agent avoiding the occasional suboptimal runs of Q-steering when using a target which favours one objective, as reported in Section 6.3. This indicates that there may be benefit in initially training on a target which provides a balanced trade-off between objectives, even if the eventual desired policy should favour one of the objectives.

\section{A Practical Demonstration of Q-Steering: Battery Control}

While Section 6 used tabular Q-learning, Q-steering is also applicable to problems where the state space is too large for tabular methods to be appropriate. To illustrate this, this section reports results obtained by applying Q-steering in conjunction with tile-coding to a simulation of controlling local battery storage for a residential photovoltaic power generation system.

Local storage allows the power generated to be 'time-shifted'to better match the household's demand profile, delivering benefits in energy purchase costs and environmental impact. A simple solution is to over-size the battery, but this has a significant capital cost - a typical long-duration battery can cost between AUS $\$ 400 / \mathrm{kWh}$ and AUS $\$ 1000 / \mathrm{kWh}[30]$. As such, the first objective is to reduce the amount of external power consumed by the household for a small battery system. An ideal storage system will dynamically match load through rapid charge and discharge response. However while many battery chemistries can supply and sink a significant amount of power for short periods, this adversely impacts on their energy capacity and lifespan, particularly when done repeatedly over long periods of time [31]. So a second, conflicting objective is to minimise the wear on the battery.

The simulation is based on a simplified battery model with linear dynamics, assuming a round-trip efficiency of $90 \%$ (with a fixed $10 \%$ loss during charging). It is also assumed that the long-term battery wear can be approximated by the amount discharged or absorbed by the battery at each time step. The power generated by the solar panels and the load consumed are derived from one year's worth of historical data from a real household. The agent acts on an hourly basis, selecting between 4 different levels of charging 
or discharging the battery, or to not use the battery at all, for a total of 9 actions. The state information consists of five continuous variables (the battery charge level and perfect forecasts of load and generation for both one and six hours ahead), so tabular methods are impractical. Instead the agent uses tile-coding [32] to represent the values for each base policy.

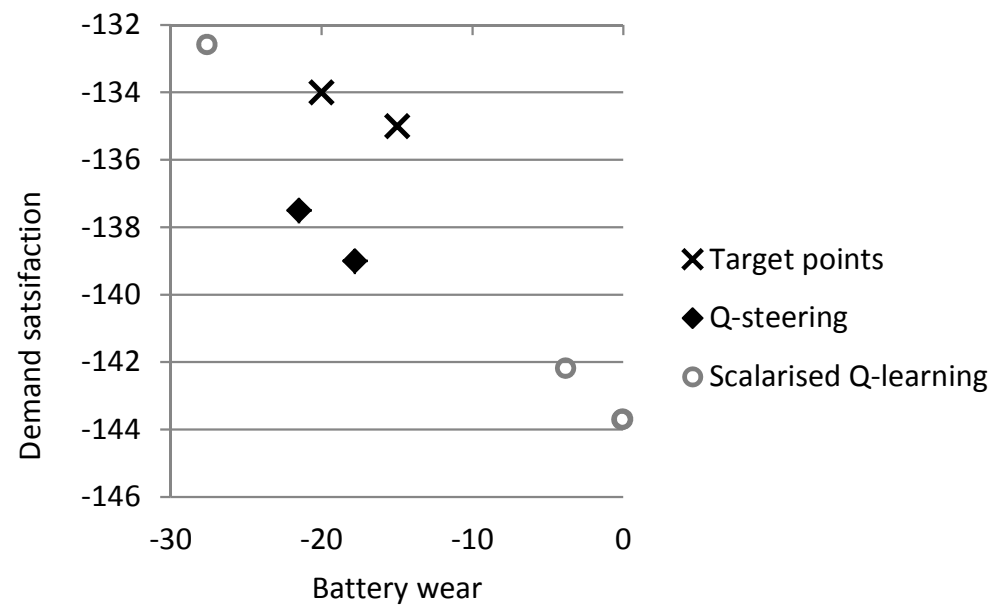

Figure 12: The approximate Pareto front found by scalarised Q-learning on the battery control task, and the results found by Q-steering using two different optimistic target points. Both objectives are measured in terms of the mean hourly value in Wh.

Figure 12 shows the final approximate Pareto front discovered by multiple runs of scalarised Q-learning (which can only find LDS policies) and Qsteering. For scalarised Q-learning the objective weights tested were evenly spaced at increments of 0.1 in the range 0..1. Some runs produced results which were Pareto-dominated, or very similar to those from other runs so for clarity these have been omitted from the figure. It was observed that even small increases in the weights assigned to the battery wear objective resulted in scalarised Q-learning converging to policies that were strongly biased towards this objective, making very little use of the battery. Therefore an approximation of the Pareto front using uniformly spaced sets of weights failed to find any policies which provided equitable trade-offs between the objectives. In contrast when target points representing such trade-offs ($15,-135)$ and $(-20,-134)$ were provided to Q-steering, the policies found corresponded quite closely to the specified targets (note that the target points were chosen optimistically and can not actually be achieved). The results achieved by Q-steering are also comparable to those produced by several 
hand-crafted policies derived from expert knowledge. Therefore the use of non-stationary policies as learnt by Q-steering has enabled a closer match to the user's desired outcomes than could be achieved using deterministic stationary MORL methods.

\section{Conclusion and Future Work}

Learning Pareto-optimal non-stationary policies is important for many MORL tasks, as these policies may be superior (in terms of Pareto-dominance) to deterministic stationary policies, and better able to match the preferred trade-off between objectives. The MDRL steering algorithm $[7,8]$ forms a non-stationary mixture of multiple deterministic base policies conditioned on the vector of rewards received so far. This paper has examined two adaptations of steering (w-steering and Q-steering) designed for learning a Paretooptimal policy, with the preference between objectives specified via a target point in reward space. w-steering is a direct adaptation of MDRL, while Qsteering incorporates a novel policy-selection heuristic designed to improve its performance, particularly for environments where the agent cannot be provided with prior knowledge about the recurrent states of the environment.

The empirical results presented show that both Pareo-optimal steering algorithms exploit the benefits of non-stationarity to substantially outperform deterministic stationary polcies, producing a reduction in error on the order of $40-100 \%$ on the benchmark problems. In addition by taking into account the current state of the environment Q-steering substantially outperforms w-steering where knowledge of the recurrent states is not available, or where there are no recurrent states. This represents a significant improvement over the original formulation of the steering approach to MORL, offering scope for application to a much broader class of problems. In addition the results reported on the episodic DST-Mixed task indicate that Q-steering also addresses the policy-identification problem of w-steering, producing slightly improved results for optimistic targets even when the recurrent state information is available.

Q-steering also supports interactive exploration of a multiobjective task by a human user. Section 7 demonstrated that the Pareto front can be estimated while Q-steering learns from an optimistic target point. This estimated front allows the user to make a more informed specification of the target, which produces an immediate change in the behaviour of the agent. 
Therefore Q-steering can be utilised by a decision-maker even with little prior knowledge of the outcomes achievable for each objective.

The ability of Q-steering to scale to more complex problems has also been demonstrated on a simulated battery control task. The state space of this task has sufficient dimensions to render tabular methods impractical, and so a form of function approximation is required to store the Q-values (in this case, tile-coding was used). Function approximation can be incorporated into Q-steering with no further alteration to the algorithm, as demonstrated by the results observed on this task, where Q-steering was able to match the specified targets much more closely than scalarised Q-learning.

While Q-steering represents a significant addition to the arsenal of MORL algorithms, a number of issues still require further investigation:

- In Section 6.3 it was observed that Q-steering failed to converge to a fully optimal policy in a small percentage of runs where the target point strongly favoured one objective. The results in Section 7 indicated this did not occur when the target was balanced with regards to both objectives, which suggests the cause is that an unbalanced target leads to some base policies being active only infrequently, resulting in their learning being slowed due to more frequent clearing of the eligibility traces. Future work should investigate whether alternative approaches to exploration (such as optimistic initialisation, or exploratory selection at policy rather than action level) may ameliorate this issue [33].

- During the experiments reported in Section 8 issues were encountered with the memory requirements of Q-steering - even with the use of tile-coding, the need to learn Q-values for many base policies leads to high memory usage compared to single-policy methods. As noted in [12] the optimal steering policy for a problem with $n$ objectives will only use a maximum of $n$ base policies. However the current formulation of Q-steering requires that policies be learnt for many different weight sets in order to ensure that the required base policies are discovered. Methods for minimising the number of base policies, such as by adapting the weights during learning [25], would be beneficial in reducing the memory overhead of Q-steering.

- The results reported in this paper use Q-learning as the algorithm for base policies. However previous research has reported divergence issues for Q-learning in conjunction with function-approximation [34]. While 
such problems were not observed during the experiments in Section 8, if Qsteering is to be effectively applied to large problems it will be necessary to use more stable off-policy learning methods as described in [35]. However this is merely an implementation issue as any off-policy temporal-difference algorithm can readily be incorporated into the Q-steering algorithm.

It should also be noted that non-stationary policies may be inappropriate in certain applications. The patient treatment scenario examined by [36] is an excellent example. A non-stationary policy which switches between one base policy which minimises symptoms but also maximises side effects and another which does the reverse may appear to produce an excellent trade-off between those objectives when rewards are averaged over multiple episodes of treating different patients. However the experience of any individual patient is unlikely to be desirable. Therefore there still exists a need to develop better algorithms for finding stationary deterministic policies for such tasks. The recent Pareto-set algorithm [37, 38] represents a promising new approach for identifying such policies.

\section{Acknowledgements}

We would like to acknowledge the support received from the CSIRO Flagship Collaboration Fund and the Australian Federal Government's Collaborative Research Network program.

\section{Appendix A. Target points used in steering experiments}

\begin{tabular}{l|l}
\hline Distance from hull & DST-Mixed \\
-1 & $(5.0939,-3.9138),(15.223,-11.9866),(18.2922,-16.7873)$ \\
-0.5 & $(5.2969,-3.4569),(15.5865,-11.6433),(18.7711,-16.6437)$ \\
0 & $(5.5,-3),(15.95,-11.3),(19.25,-16.5)$ \\
0.5 & $(5.7031,-2.5431),(16.3135,-10.9567),(19.7289,-16.3563)$ \\
1 & $(5.9061,-2.0862),(16.677,-10.6134),(20.2078,-16.2127)$ \\
1.5 & $(6.1092,-1.6293),(17.0405,-10.2701),(20.6867,-16.069)$ \\
2 & $(6.3123,-1.1724),(17.404,-9.9268),(21.1657,-15.9253)$ \\
\hline
\end{tabular}

Table A.4: The target points used for DST-Mixed. Negative distances indicate pessimistic targets, 0 indicates neutral targets, and positive distances correspond to optimistic targets. Values have been rounded to 4 decimal places for purposes of display. 


\begin{tabular}{l|l}
\hline Distance from hull & $\begin{array}{l}\text { Linked Rings } \\
-1\end{array}$ \\
$-(-0.7071,1.2929),(0.2929,0.2929),(1.2929,-0.7071)$ \\
-0.5 & $(-0.3536,1.6464),(0.6464,0.6464),(1.6464,-0.3536)$ \\
0 & $(0,2),(1,1),(2,0)$ \\
0.5 & $(0.3536,2.3536),(1.3536,1.3536),(2.3536,0.3536)$ \\
1 & $(0.7071,2.7071),(1.7071,1.7071),(2.7071,0.7071)$ \\
1.5 & $(1.0607,3.0607),(2.0607,2.0607),(3.0607,1.0607)$ \\
2 & $(1.4142,3.4142),(2.4142,2.4142),(3.4142,1.4142)$ \\
\hline
\end{tabular}

Table A.5: The target points used for Linked Rings

\begin{tabular}{l|l}
\hline Distance from hull & Non-Recurrent Rings \\
-1 & $(-0.9571,0.5429),(-0.2071,-0.2071),(0.5429,-0.9571)$ \\
-0.5 & $(-0.6036,0.8964),(0.1464,0.1464),(0.8964,-0.6036)$ \\
0 & $(-0.25,1.25),(0.5,0.5),(1.25,-0.25)$ \\
0.5 & $(0.1036,1.6036),(0.8536,0.8536),(1.6036,0.1036)$ \\
1 & $(0.4571,1.9571),(1.2071,1.2071),(1.9571,0.4571)$ \\
1.5 & $(0.8107,2.3107),(1.5607,1.5607),(2.3107,0.8107)$ \\
2 & $(1.1642,2.6642),(1.9142,1.9142),(2.6642,1.1642)$ \\
\hline
\end{tabular}

Table A.6: The target points used for Non-Recurrent Rings

[1] A. Castelletti, G. Corani, A. Rizzolli, R. Soncini-Sessa, E. Weber, Reinforcement learning in the operational management of a water system, in: IFAC Workshop on Modeling and Control in Environmental Issues, 2002, pp. 325-330.

[2] J. Oksanen, J. Lundén, V. Koivunen, Reinforcement learning based sensing policy optimization for energy efficient cognitive radio networks, Neurocomputing 80 (2012) 102-110.

[3] Y.-J. Liu, Y. Gao, S. Tong, Y. Li, Fuzzy approximation-based adaptive backstepping optimal control for a class of nonlinear discrete-time systems with dead-zone (2015).

[4] T. Brys, K. Van Moffaert, K. Van Vaerenbergh, A. Nowé, On the behaviour of scalarization methods for the engagement of a wet clutch, in: Proceedings of the 12th International Conference on Machine Learning and Applications (ICMLA 2013), IEEE, 2013. 
[5] D. Roijers, P. Vamplew, S. Whiteson, R. Dazeley, A survey of multiobjective sequential decision-making, Journal of Artificial Intelligence Research 48 (2013) 67-113.

[6] P. Vamplew, R. Issabekov, R. Dazeley, C. Foale, Reinforcement learning of pareto-optimal multiobjective policies using steering, in: AI 2015: Advances in Artificial Intelligence, Springer, 2015, pp. 596-608.

[7] S. Mannor, N. Shimkin, The steering approach for multi-criteria reinforcement learning, in: Neural Information Processing Systems, 2001, pp. 1563-1570.

[8] S. Mannor, N. Shimkin, A geometric approach to multi-criterion reinforcement learning, Journal of Machine Learning Research 5 (2004) $325-360$.

[9] P. Vamplew, R. Dazeley, A. Berry, E. Dekker, R. Issabekov, Empirical evaluation methods for multiobjective reinforcement learning algorithms, Machine Learning 84 (2011) 51-80.

[10] C. Shelton, Importance Sampling for Reinforcement Learning with Multiple Objectives, AI Technical Report 2001-003, MIT, 2001.

[11] K. Chatterjee, R. Majumdar, T. Henzinger, Markov decision processes with multiple objectives, in: Proceedings of the 23rd Annual conference on Theoretical Aspects of Computer Science, STACS'06, SpringerVerlag, Berlin, Heidelberg, 2006, pp. 325-336.

[12] P. Vamplew, R. Dazeley, E. Barker, A. Kelarev, Constructing stochastic mixture policies for episodic multiobjective reinforcement learning tasks, in: AI'09: The 22nd Australasian Conference on Artificial Intelligence, 2009, pp. 340-349.

[13] S. Parisi, M. Pirotta, N. Smacchia, L. Bascetta, M. Restelli, Policy gradient approaches for multi-objective sequential decision making, in: 2014 International Joint Conference on Neural Networks (IJCNN), IEEE, 2014, pp. 2323-2330.

[14] H. Handa, Solving multi-objective reinforcement learning problems by EDA-RL - acquisition of various strategies, in: Proceedings of the Ninth 
Internatonal Conference on Intelligent Sysems Design and Applications, 2009, pp. 426-431.

[15] H. Soh, Y. Demiris, Evolving policies for multi-reward partially observable Markov decision processes (MR-POMDPs), in: GECCO'11 Proceedings of the 13th Annual Conference on Genetic and Evolutionary Computation, 2011, pp. 713-720.

[16] M. E. Taylor, S. Whiteson, P. Stone, Temporal difference and policy search methods for reinforcement learning: An empirical comparison, in: Proceedings of the National Conference on Artificial Intelligence, volume 22, Menlo Park, CA; Cambridge, MA; London; AAAI Press; MIT Press; 1999, 2007, p. 1675.

[17] S. Kalyanakrishnan, P. Stone, An empirical analysis of value functionbased and policy search reinforcement learning, in: Proceedings of The 8th International Conference on Autonomous Agents and Multiagent Systems-Volume 2, International Foundation for Autonomous Agents and Multiagent Systems, 2009, pp. 749-756.

[18] S. Whiteson, M. E. Taylor, P. Stone, Critical factors in the empirical performance of temporal difference and evolutionary methods for reinforcement learning, Autonomous Agents and Multi-Agent Systems 21 (2010) 1-35.

[19] D. M. Roijers, S. Whiteson, F. A. Oliehoek, Computing convex coverage sets for multi-objective coordination graphs, Algorithmic Decision Theory, Springer, 2013, pp. 309-323.

[20] J. Karlsson, Learning to Solve Multiple Goals, Ph.D. thesis, University of Rochester, 1997.

[21] Y. Guo, A. Zeman, R. Li, A reinforcement learning approach to setting multi-objective goals for energy demand management, International Journal of Agent Technologies and Systems 1 (2009) 55-70.

[22] L. Ferreira, R. Bianchi, C. Ribeiro, Multi-agent multi-objective reinforcement learning using heuristically accelerated reinforcement learning, in: 2012 Brazilian Robotics Symposium and Latin American Robotics Symposium, 2012, pp. 14-20. 
[23] P. Vamplew, J. Yearwood, R. Dazeley, A. Berry, On the limitations of scalarisation for multi-objective reinforcement learning of Pareto fronts, in: AI'08: The 21st Australasian Joint Conference on Artificial Intelligence, Springer, 2008, pp. 372-378.

[24] L. Barrett, S. Narayanan, Learning all optimal policies with multiple criteria, in: Proceedings of the 25th International Conference on Machine Learning, ACM, New York, NY, USA, 2008, pp. 41-47. URL: http://doi.acm.org/10.1145/1390156.1390162. doi:10.1145/1390156.1390162.

[25] K. Van Moffaert, T. Brys, A. Chandra, L. Esterle, P. R. Lewis, A. Nowé, A novel adaptive weight selection algorithm for multi-objective multiagent reinforcement learning, in: Neural Networks (IJCNN), 2014 International Joint Conference on, IEEE, 2014, pp. 2306-2314.

[26] P. Raicevic, Parallel reinforcement learning using multiple reward signals, Neurocomputing 69 (2006) 2171-2179.

[27] D. Lizotte, Multi-objective markov decision processes for decision support, in: The Multi-disciplinary Conference on Reinforcement Learning and Decision Making, 2015.

[28] R. Akrour, M. Schoenauer, M. Sebag, Preference-based policy learning, in: Joint European Conference on Machine Learning and Knowledge Discovery in Databases, Springer, 2011, pp. 12-27.

[29] J. Fürnkranz, E. Hüllermeier, W. Cheng, S.-H. Park, Preference-based reinforcement learning: a formal framework and a policy iteration algorithm, Machine learning 89 (2012) 123-156.

[30] T. S. Brinsmead, P. Graham, J. Hayward, E. L. Ratnam, L. Reedman, Future Energy Storage Trends: An Assessment of the Economic Viability, Potential Uptake and Impacts of Electrical Energy Storage on the NEM 2015-2035, Technical Report EP155039, CSIRO, 2015.

[31] K. Cavanagh, J. K. Ward, S. Behrens, A. I. Bhatt, E. L. Ratnam, E. Oliver, J. Hayward, Electrical energy storage: technology overview and applications, Technical Report EP154168, CSIRO, 2015. 
[32] R. Sutton, Generalization in reinforcement learning: Successful examples using sparse coarse coding, Advances in neural information processing systems (1996) 1038-1044.

[33] P. Vamplew, R. Dazeley, C. Foale, A novel exploration method for multiobjective reinforcement learning, Neurocomputing (this issue) (2016).

[34] D. Precup, R. Sutton, S. Dasgupta, Off-policy temporal-difference learning with function approximation, in: ICML, 2001, pp. 417-424.

[35] R. Sutton, C. Szepesvári, H. Maei, A convergent O(n) algorithm for offpolicy temporal-difference learning with linear function approximation (2009).

[36] D. J. Lizotte, M. Bowling, S. A. Murphy, Efficient reinforcement learning with multiple reward functions for randomized clinical trial analysis, in: 27th International Conference on Machine Learning, 2010, pp. 695-702.

[37] K. Van Moffaert, M. M. Drugan, A. Nowé, Learning sets of pareto optimal policies, in: Thirteenth International Conference on Autonomous Agents and Multiagent Systems-Adaptive Learning Agents Workshop (ALA), 2014.

[38] K. Van Moffaert, A. Nowé, Multi-objective reinforcement learning using sets of pareto dominating policies, Journal of Machine Learning Research 15 (2014) 3483-3512. 